Check for updates

Cite this: RSC Adv., 2017, 7, 23065

Received 30th December 2016

Accepted 6th March 2017

DOI: $10.1039 / c 6 r a 28843 j$

rsc.li/rsc-advances

\section{Optimizing the balance between stiffness and flexibility by tuning the compatibility of a poly(lactic acid)/ethylene copolymer}

\begin{abstract}
Liang-liang Zhao, ${ }^{a} J u a n-j u a n ~ S u,{ }^{* a b}$ Jian Han, ${ }^{\star a b}$ Bin Zhang ${ }^{a}$ and $\mathrm{Lu} \mathrm{Ou}^{\mathrm{a}}$
The brittleness of poly(lacticacid) (PLA) is a major drawback for its wide application. In this study, a superflexible PLA material was prepared by reactive blending of PLA with poly(ethylene-glycidyl methacrylate) (EGMA), and the weight ratio was fixed at $95: 5$. The investigation showed that the PLA/EGMA blend had limited interfacial compatibility. The strong interfacial interaction and fine dispersion of EGMA particles were obtained by using initiator DCP, which it had been demonstrated could accelerate the reaction efficiency between PLA and EGMA. The elongation at break of the PLA/EGMA/DCP blend was remarkably improved, about 58 folds that of neat PLA. More importantly, its outstanding tensile strength and transparency were definitely maintained as that of neat PLA. Our work provides an effective method to largely improve the flexibility of PLA without sacrificing its stiffness and transparency, which is very important for the wide application of PLA materials.
\end{abstract}

\section{Introduction}

With the increasing awareness of environmental concerns and sustainability, poly(lactic acid) (PLA) has attracted much attention in recent years as an environmentally degradable polymer which is derived from renewable resources. ${ }^{1-3}$ Besides, PLA possesses good mechanical strength, easy processability and excellent transparency. It has potential applications in biomedical devices, packaging and the automotive industry. ${ }^{4-6}$ Currently, PLA has been thought to be the most promising alternative to traditional commodity plastics. However, as a major drawback of PLA, the inherent brittleness (as evidenced by relatively low values of the tensile strain at break, tensile toughness and impact strength) caused by the low entanglement density (Ve) and the chain stiffness has limited its various end-user applications. ${ }^{7,8}$

In recent years, many efforts have been made to improve the toughness of PLA, and various strategies have been developed. Rigid particles, such as montmorillonite, multi-walled carbon nanotubes, etc, were used as toughening agents, while the toughening efficiency was very limited., ${ }^{\mathbf{9} 10}$ Blending PLA with flexible polymers or rubbers is known as one of the most common and effective strategy. Degradable and non-degradable polymers, such as poly(butylene adipate-co-terephthalate) (PBAT), ${ }^{11}$ poly(butylenesuccinate) (PBS), ${ }^{12}$ poly(3-caprolactone) (PCL), ${ }^{13}$ EVA, ${ }^{14}$ castor oil, ${ }^{15}$ polyurethane (PU), ${ }^{16}$ and $\mathrm{EGMA}^{17-20}$ etc, have been used to compound with PLA for improving its impact strength or

${ }^{a}$ College of Materials and Textiles, Zhejiang Sci-Tech University, Hangzhou 310018, People's Republic of China. E-mail: sujuanjuan@zstu.edu.cn

${ }^{b}$ The Key Lab of Industrial Textile Material and Manufacturing Technology, Hangzhou 310018, Zhejiang Provence, People's Republic of China flexibility by direct mechanical blending. It is evidently that the manifested brittleness of these PLA based blends was mainly ascribed to the incompatibility of the two polymers, leading to a lack of adhesion between the components and subsequently resulting in unstable cracking. ${ }^{21,22}$ As we all know, EGMA is a reactive elastomer containing epoxide groups, which has high reactivity toward carboxyl and hydroxyl functional groups of PLA. Oyama $^{17}$ prepared a super-tough PLA materials by reactive blending PLA with poly(ethylene-glycidyl methacrylate) (EGMA). The elongation at break of PLA/EGMA blend was 40 times higher than that of neat PLA, and the ratio of PLA/EGMA was 80/20. However, the tensile modulus and yield strength of 80PLA/ 20EGMA blend decreased about $27.2 \%$ and $37.8 \%$, respectively. Liu et al. ${ }^{18,19}$ fabricated a super toughened PLA ternary blend containing ethylene/ $n$-butyl acrylate/glycidyl methacrylate (EBAGMA) and zinc ionomer of ethylene/methacrylic acid (EMAA-Zn) by simultaneous interfacial compatibility and dynamic vulcanization. It was found that the zinc ions catalyzed the cross-linking of epoxy-containing elastomer, meantime, promoted the reactive compatibility between PLA and elastomer. Super toughened PLA material was obtained, nevertheless, the tensile strength and tensile modulus decreased about $40.8 \%$ and $35.5 \%$, respectively. Dong et $a .^{20}$ reported PLA alloys with the nanometer rubber domains $(<100 \mathrm{~nm})$ by reactive blending PLA with ethylene-coacrylic ester-co-glycidyl methacrylate (E-AE-GMA) (80/20, w/w), and the flexibility was significantly improved, coincidentally, tensile strength and tensile modulus decreased about $36.2 \%$ and $62.6 \%$, respectively. It is still a challenge to prepare PLA materials with good balance of toughness and stiffness.

Typically, super-tough behavior could be achieved when the optimum elastomer content about $20 \mathrm{wt} \%$ was added, and that 
means dramatic sacrifice of process-ability and stiffness. ${ }^{23,24}$ It is generally accepted that, despite cavitation of elastomer particles does contribute a little to the dissipation of fracture energy, the cavitated particles can relax the locally triaxial stress and then initiate crazing and/or matrix shear yielding around them. ${ }^{25,26}$ Matrix shear yielding is a more effective energy dissipation manner, compared to crazing, and the key to achieve ideal toughening effect is matrix shear yielding. ${ }^{27}$ Therefore, reducing the average size of elastomer particles and promoting the interfacial adhesion are crucial to lower down the optimum elastomer loading for matrix shear yielding.

In this paper, we aimed to prepare PLA/EGMA blend with good balance of flexibility and stiffness. The weight ratio of PLA/ EGMA was fixed at 95/5, and a free radical initiator of dicumyl peroxide (DCP) was introduced to modulate the interfacial compatibility of EGMA with PLA matrix. ${ }^{28-30}$ The effects of DCP on compatibilization of PLA/EGMA blend and consequently the microstructure of the dispersed particles were systematically investigated. This report focuses on the importance of interface control in the preparation of elastomer toughened PLA blends, in which finer dispersion morphology and stronger interfacial adhesion can successfully lower down the optimum elastomer loading for significantly improving the flexibility, and finally high-performance PLA/EGMA blend with excellent flexibility was achieved without stiffness sacrificing.

\section{Experimental section}

\subsection{Materials}

The materials of PLA and EGMA are commercially available. PLA (4032D, Nature Works LLC) with high stereo regularity (1.2$1.6 \% \mathrm{D}$-isomer lactide), exhibits a density of $1.25 \mathrm{~g} \mathrm{~cm}^{-3}$. The weight-averaged molecular weight $\left(M_{\mathrm{W}}\right)$ and polydispersity are $207 \mathrm{kDa}$ and 1.74, respectively. EGMA (glycidyl methacrylate), Lotader® AX8900 was obtained from ARKEMA (Puteaux, France). It is a terpolymer of ethylene-methyl acrylate andglycidyl-methacrylate (E-MA-GMA). According to the datasheet of the material, methyl acrylate and GMA contents are 24 and $8 \mathrm{wt} \%$, respectively. The dicumyl peroxide (DCP) was supplied by Chen Guan Co. (Sichuan, China).

\subsection{Sample preparation}

Blends of PLA/EGMA/DCP (PLA/EGMA was fixed at 95/5) with varying DCP content were prepared using a Haake mixer (XSS300 , USA) at a temperature of $190{ }^{\circ} \mathrm{C}$ and rotor speed of $60 \mathrm{rpm}$ for $8 \mathrm{~min}$. After making droplets, the pellets were compression molded at $190{ }^{\circ} \mathrm{C}$ for $5 \mathrm{~min}$ under $10 \mathrm{MPa}$ into standard specimens for mechanical and rheology tests. Before melt mixing and compression molding, all the materials were dried in a vacuum oven at $50^{\circ} \mathrm{C}$ for at least $12 \mathrm{~h}$. To comparison, neat PLA was also processed at the same condition.

\subsection{Mechanical testing}

Tensile testing was performed with a SANS universal tensile testing machine at a cross-head speed of $5 \mathrm{~mm} \min ^{-1}$, according to ISO 527-3 standard. For each sample, the measurements were carried out at room temperature $\left(23^{\circ} \mathrm{C}\right)$ and the results obtained were averaged over at least five specimens. Before testing, the samples were preconditioned in 50\% relative humidity at room temperature for $48 \mathrm{~h}$. The notched Izod impact strength of the specimens was measured with a VJ40 Izod machine according to ASTM: D256-10. Before measurement, a $45^{\circ} \mathrm{V}$ shaped notch was made (depth $2 \mathrm{~mm}$ ). The measurement was carried out at room temperature and the average value reported was derived from at least five specimens.

\subsection{Differential scanning calorimetry (DSC)}

The calorimetric analysis was conducted using a Perkin-Elmer pyris-I DSC in a dry nitrogen atmosphere, calibrated by indium. For each measurement, around $5 \mathrm{mg}$ sample was heated from 25 to $200{ }^{\circ} \mathrm{C}$ at a heating rate of $10{ }^{\circ} \mathrm{C} \mathrm{min}{ }^{-1}$. The crystallinity of PLA $\left(X_{\mathrm{c}}\right)$ was estimated from first heating cycle using the following equation: ${ }^{18,19}$

$$
X_{\mathrm{c}}=\frac{\Delta H_{\mathrm{m}}-\Delta H_{\mathrm{c}}}{w_{\mathrm{f}} \Delta H_{\mathrm{m}}^{0}} \times 100 \%
$$

where $X_{\mathrm{c}}=$ mass crystallinity, $\Delta H_{\mathrm{m}}^{0}=$ heat of fusion of $100 \%$ crystalline PLA (93.7 $\mathrm{J} \mathrm{g}^{-1}$ from the ATHAS databank), $\Delta H_{\mathrm{m}}=$ melt enthalpy of PLA, $\Delta H_{\mathrm{c}}=$ (cold) crystallisation enthalpy of PLA, $w_{\mathrm{f}}=$ PLA weight fraction in the blend.

\subsection{Fourier transform infrared spectroscopy (FT-IR)}

The absorption spectra were recorded using a Thermo Nicolet FTIR spectrometer with a resolution of $4 \mathrm{~cm}^{-1}$ and 32 scans. For each PLA/EGMA/DCP blend, the test specimens were firstly cut into films with $\sim 20 \mu \mathrm{m}$ in thickness by a Leica RM2245 microtome from the center of compression-molded bars. These films were extracted with 1 , 4-dioxane at $120{ }^{\circ} \mathrm{C}$ under stirring for 10 days to selectively remove free PLA thoroughly, then the extracted films were used for the FT-IR test. The absorption spectra of neat PLA and EGMA as a reference were also characterized.

\subsection{Scanning electron microscope (SEM)}

The phase morphology of PLA/EGMA/DCP blend was characterized with an FEI inspect F field emission scanning electron microscope at an accelerating voltage of $5.0 \mathrm{kV}$. Samples for dispersed phase morphology observation were cryogenically fractured. Tensile fractured surfaces were also characterized with SEM. Before SEM characterizations, all the surfaces were sputter-coated with a gold layer.

\subsection{Rheological measurements}

The rheological measurements were performed on a controlled stress rheometer (Malvern Instruments Ltd., UK). Testing sample disks with a thickness of $1.2 \mathrm{~mm}$ and a diameter of 25 $\mathrm{mm}$ were prepared by compression molding of the samples at $190{ }^{\circ} \mathrm{C}$ for $3 \mathrm{~min}$. The rheological properties of samples were carried out by small-amplitude oscillatory shear frequency dependence $($ strain $=1 \%$ ) measurements within a frequency range of 0.03 to $100 \mathrm{rad} \mathrm{s}^{-1}$. All these measurements were 
conducted under a nitrogen atmosphere to minimize oxidative degradation of the samples.

\section{Results and discussion}

\subsection{Mechanical properties}

Whether DCP is beneficial for improving the toughness of PLA could be taken into account. The notched Izod impact strengths of neat PLA and its blends are presented in Table 1. The impact strength of 95PLA/5EGMA blend is almost as the same of neat PLA. However, it is gradually improved with the increasing content of DCP, indicating that DCP plays a very important role in promoting the toughening efficiency of EGMA, and thus can effectively toughen the PLA matrix.

Typical stress-strain curves of pure PLA and its blends are shown in Fig. 1(a), and the detailed average values of the mechanical properties of various samples are summarized in Table 1. As shown in Fig. 1(a), pure PLA shows a typical curve of brittle fracture with the elongation at break of $5.2 \%$, however, the tensile strength and modulus is as high as $66 \mathrm{MPa}$ and $2001 \mathrm{MPa}$, respectively. The elongation at break is slightly increased to $10 \%$ upon incorporation of $5 \mathrm{wt} \%$ EGMA. However, it is very impressed that $160 \%$ of the elongation at break is observed with the incorporation of only $0.05 \mathrm{phr}$ DCP. As the content of DCP increases up to $0.1 \mathrm{phr}$, the elongation at break is further increased to $290 \%$, which is about 58 times higher than that of pure PLA. Thereafter, the elongation at break reaches a plateau with the continue addition of DCP content. As distinctly shown in Fig. 1(b), both the tensile strength and tensile modulus, for not merely PLA/EGMA but also PLA/EGMA/ DCP blends, are almost the same as pure PLA. This is probably due to the lower content of soft EGMA elastomer and samplepreparation method. In our experiment, DCP was firstly mixed with EGMA and then melting blended with PLA. It is proposed that DCP was mainly used for accelerating the compatibilization reaction, rather than resulting the serious crosslinking or degradation of PLA matrix. Our speculation had also been confirmed by the GPC experimental results (results are not shown here). Finally, PLA/EGMA/DCP blends with excellent flexibility have been successfully fabricated and more importantly, a good balance in flexibility and stiffness has been realized. This is very important for the industrial application of PLA materials.

\subsection{Morphological structure}

The dispersed phase morphology of the examined blend systems was investigated with SEM experiments. Fig. 2 shows the SEM micrographs of cryogenically fractured morphology of 95PLA/5EGMA and 95PLA/5EGMA/0.1DCP blends. It can be seen that all the above blends exhibit a phase-separated morphology with elastomer particles dispersed in PLA matrix. The image (Fig. 2(a)) presents the binary 95PLA/5EGMA blend, which displays morphologies with average particle sizes ranging from $300 \mathrm{~nm}$ to $550 \mathrm{~nm}$, and EGMA particles are well immersed in the PLA matrix. This sub-micro scale of dispersed particle diameter ascribes from the relatively good interfacial adhesion between PLA and EGMA. The epoxide groups of EGMA are supposed to react with carboxyl and hydroxyl functional groups of PLA. It is interesting to find that, with the addition of only 0.1 phr DCP, the diameter of the uniformly dispersed EGMA particles decrease remarkably into a range from $100 \mathrm{~nm}$ to $220 \mathrm{~nm}$, and the interface turns obscure (Fig. 2(b)), indicating

Table 1 Mechanical properties of PLA and its blends

\begin{tabular}{|c|c|c|c|c|}
\hline Samples & $\begin{array}{l}\text { Elongation at } \\
\text { break }(\%)\end{array}$ & $\begin{array}{l}\text { Tensile strength } \\
\text { (MPa) }\end{array}$ & $\begin{array}{l}\text { Tensile } \\
\text { modulus (MPa) }\end{array}$ & $\begin{array}{l}\text { Impact strength } \\
\left(\mathrm{kJ} \mathrm{m}^{-2}\right)\end{array}$ \\
\hline PLA & $5.34 \pm 0.97$ & $66 \pm 4.43$ & $2001.15 \pm 65.26$ & $3.95 \pm 0.26$ \\
\hline 95PLA/5EGMA/0.05DCP & $160 \pm 13.25$ & $68 \pm 1.85$ & $1999.93 \pm 24.35$ & $5.06 \pm 0.21$ \\
\hline 95PLA/5EGMA/0.1DCP & $290 \pm 21.45$ & $67 \pm 2.21$ & $1954.24 \pm 54.36$ & $5.54 \pm 0.16$ \\
\hline 95PLA/5EGMA/0.2DCP & $270 \pm 25.24$ & $67 \pm 1.14$ & $1936.15 \pm 47.45$ & $5.87 \pm 0.18$ \\
\hline
\end{tabular}
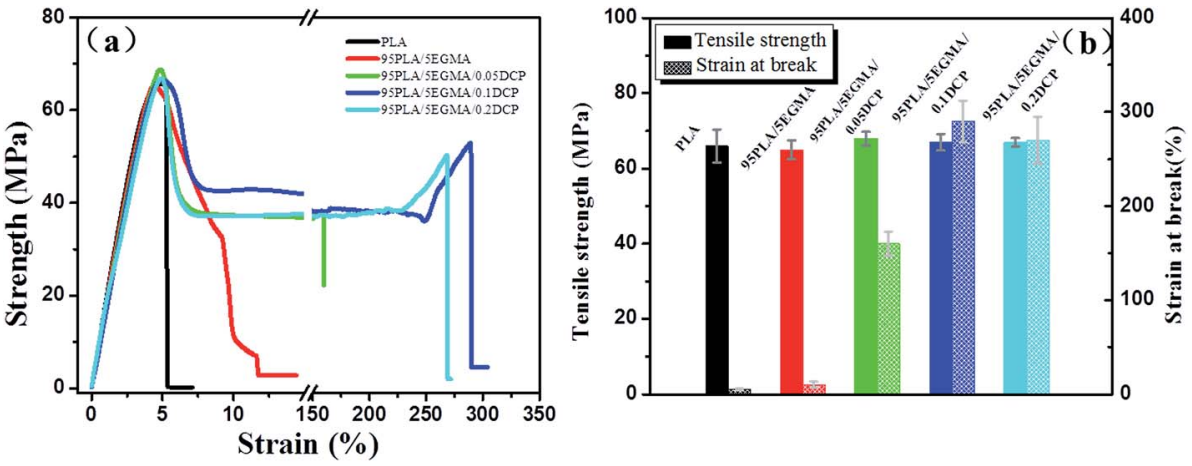

Fig. 1 Tensile properties for PLA and its blends: (a) typical tensile stress-strain curves and (b) bar graph of tensile strength and strain at break. 

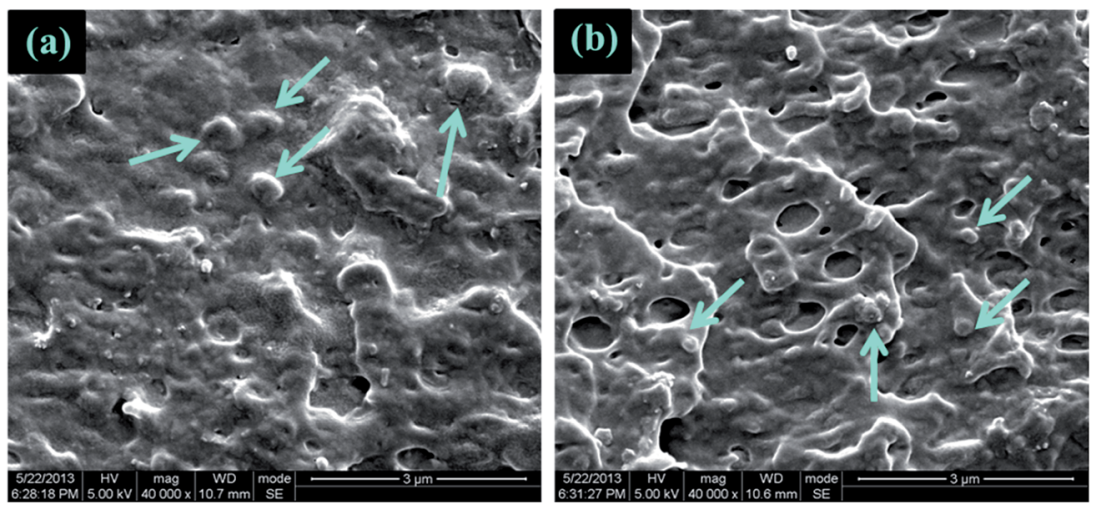

Fig. 2 SEM micrographs of samples: (a) 95PLA/5EGMA, and (b) 95PLA/5EGMA/0.1DCP

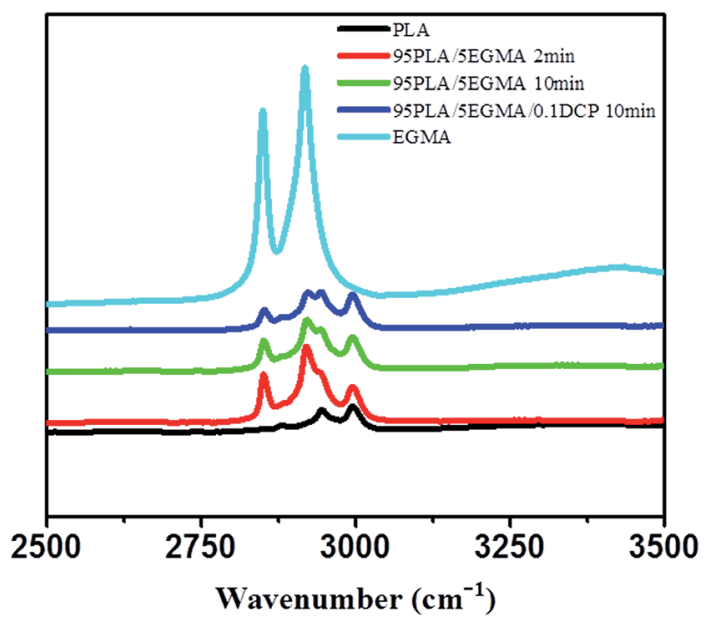

Fig. 3 FTIR absorption spectras of neat PLA and its blends.

excellent interfacial adhesion between EGMA and PLA matrix is obtained by using DCP as the catalyzer.

\subsection{Interfacial interaction between EGMA and PLA matrix}

The above SEM morphological structure displays well compatibilization effect of DCP upon PLA/EGMA blend. In order to further explore the compatibilization mechanism, FT-IR was utilized to analyze the reactive compatibilization between EGMA and PLA matrix. Fig. 3 shows the absorption spectra of each component and PLA/EGMA/DCP blends in the range of $3500-2500 \mathrm{~cm}^{-1}$. PLA exhibits absorption peaks at $2995 \mathrm{~cm}^{-1}$ and $2945 \mathrm{~cm}^{-1}$, respectively, which are attributed to the stretching vibrations of $=\mathrm{C}-\mathrm{H}$ groups and $\mathrm{CH}_{3}$ groups. For EGMA, the absorption peaks of $2930 \mathrm{~cm}^{-1}$ and $2850 \mathrm{~cm}^{-1}$ are ascribed to the asymmetric stretching and stretching vibrations of alkyl chain $\mathrm{CH}_{2}$, respectively. FT-IR spectra of the residues, which are obtained by thoroughly extracting PLA from the 95PLA/5EGMA and 95PLA/5EGMA/0.1DCP blends, are also manifested in Fig. 3. The solvent (i.e., 1,4-dioxane) can only selectively dissolve PLA, therefore, the residues is believed to include only EGMA component. Noticeably, the absorption peaks of PLA, at $2995 \mathrm{~cm}^{-1}$ and $2945 \mathrm{~cm}^{-1}$, are visible in the residues of 95PLA/5EGMA blend by melt blending for 2 or $10 \mathrm{~min}$. This indicates that the in situ formed graft copolymer is resulted by interfacial reacting of EGMA and PLA. It is also evidenced in the literature that GMA-functionalized copolymers could react with hydroxyl and/or carboxyl end groups of polyesters under melt processing conditions. ${ }^{12,31}$ As shown in Fig. 3, the spectrum of residues of the dioxane-extracted 95PLA/ 5EGMA/0.1DCP blend is closely identical to that of residues of 95PLA/5EGMA blend. The extent of the compatibilization reactions can be approximately described by the amount of grafted PLA per unit of EGMA, as measured by the ratio of absorption peak area at $2945 \mathrm{~cm}^{-1}$ to that at $2930^{-1}$, i.e., $A_{\mathrm{PLA}} /$ $A_{\mathrm{EGMA}}$. As shown in Table 2, the ratio of $A_{\mathrm{PLA}} / A_{\mathrm{EGMA}}$ increases with the increasing melt blending time (reacting time) from 2 to $10 \mathrm{~min}$, and further increases with the addition of $0.1 \mathrm{phr}$ DCP. The blend of 95PLA/5EGMA/0.1DCP shows much higher value of $A_{\mathrm{PLA}} / A_{\mathrm{EGMA}}$, indicating more pronounced interfacial compatibilization effect. DCP decompose to R $\infty$ radicals, which abstract hydrogen from both PLA and EGMA to yield free radicals. The mechanism of accelerated reaction between PLA and EGMA by using DCP is proposed to similar with the work of Semba et al. ${ }^{32}$

In order to further investigate the interfacial adhesion between PLA and EGMA, rheological tests were also carried out. Shear frequency dependence of the complex viscosity and storage modulus for neat PLA and its blends are shown in Fig. 4. Evidently, the complex viscosity is remarkably increased with the addition of EGMA, and shows a further enhancement with the increasing content of DCP. In addition, an increase of the storage modulus with the addition of EGMA and DCP can also be observed. In polymer blends, the increase of complex

Table 2 FT-IR absorption peak area of PLA/EGMA and PLA/EGMA/ DCP blend

\begin{tabular}{llll}
\hline Samples & $\begin{array}{l}A_{\mathrm{PLA}_{\mathrm{CH}}} \\
\left(2945 \mathrm{~cm}^{-1}\right)\end{array}$ & $\begin{array}{l}A_{\mathrm{EGMA}-\mathrm{AsCH}_{2}} \\
\left(2920 \mathrm{~cm}^{-1}\right)\end{array}$ & $\begin{array}{l}\text { Ratio of } \\
A_{2945} / A_{2920}\end{array}$ \\
\hline PLA/EGMA-2 min & 0.993 & 1.427 & 0.6958 \\
PLA/EGMA-10 min & 1.504 & 1.141 & 1.318 \\
PLA/EGMA/0.1DCP-10 min & 1.879 & 0.825 & 2.278
\end{tabular}



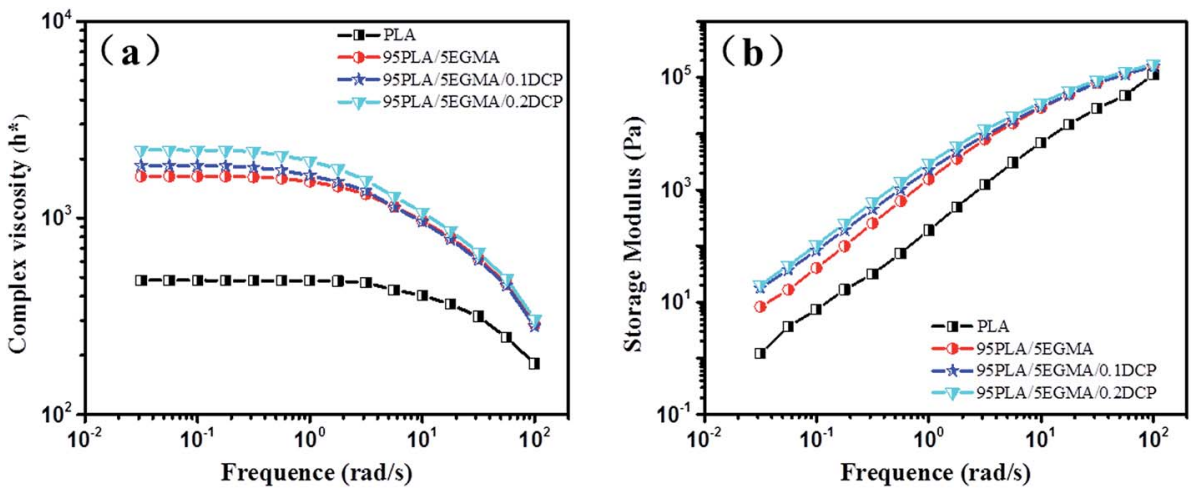

Fig. 4 Complex viscosity (a) and storage modulus (b) of neat PLA and its blend as a function of oscillatory frequency.

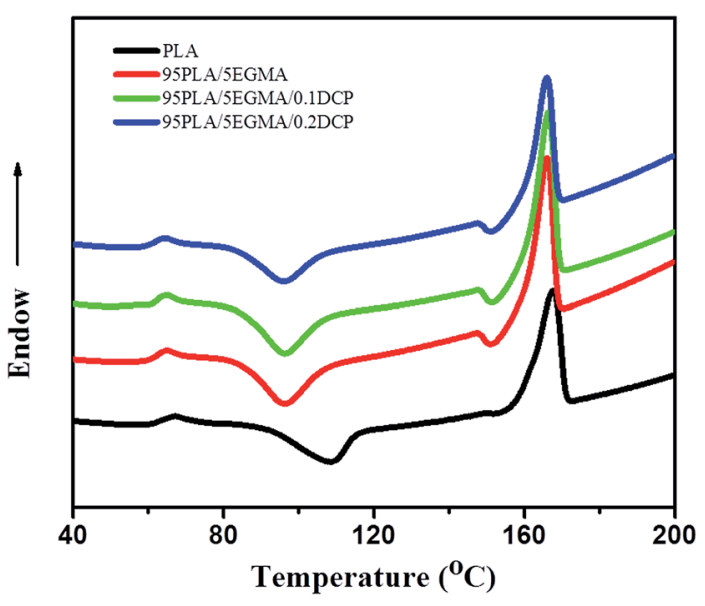

Fig. 5 DSC heating curves for the as-compression molded samples of neat PLA and its blends.

viscosity at low frequencies can be ascribed to a relaxation process of the minor phase when slightly deformed, ${ }^{33}$ and it can be used to evaluate the interfacial tension by using emulsion models. ${ }^{34-36}$ The above mentioned increase of complex viscosity demonstrates again the good interfacial adhesion of PLA/ EGMA, and it has been further enhanced by using DCP as catalyzer.

\subsection{Crystallinity of elastomer toughened PLA}

The crystallinity of PLA matrix was reported to have a significant effect on the toughness of elastomer toughened PLA blends. ${ }^{15,33}$ DSC experiments were conducted to examine the intrinsic crystallinity of PLA matrix for some typical specimens. Fig. 5 presents the first heating endothermic transition of pure PLA and its blends, including glass transition, cold-crystallization and melting transition. The results of this first heating run reflect the crystalline state present in the molded samples, which is affected by the thermal and mechanical history imposed during the molding process. It is shown that the glasstransition temperature $\left(T_{\mathrm{g}}\right)$ of PLA matrix in any blend is identical to that of pure PLA. To further explore the influence of DCP to the $T_{\mathrm{g}}$ of PLA and EGMA, DMA (results are not shown here) experiment had also been conducted. The results are coordinated to that of DSC, namely, DCP has almost no influence to the $T_{\mathrm{g}}$ of PLA and EGMA. The cold-crystallization temperature of PLA matrix for PLA/EGMA and PLA/EGMA/DCP blends is decreased about $10{ }^{\circ} \mathrm{C}$ in comparison to pure PLA, which is primarily due to the nucleating effect of EGMA particles. While the incorporation of EGMA, locally activated chain mobility at interface results in an accelerating effect of PLA crystallization. ${ }^{37}$ The cold crystallization and melting enthalpies as well as the calculated crystallinity of neat PLA and its blends are summarized in Table 3. It is evidently that the crystallinity of PLA is identical for the blends with EGMA or with the further addition of DCP.

\subsection{Toughening mechanism}

To explore the mechanism for significantly increased flexibility generated by the addition of DCP, the tensile fracture surface of pure PLA and its blends were characterized by SEM experiments. As shown in Fig. $6\left(a^{\prime}\right)$ and $\left(b^{\prime}\right)$, the extremely smooth tensile fracture surface, representing for pure PLA and 95PLA/ 5EGMA blend, respectively, displays a typical brittle fracture through unstable crazing. However, it clearly demonstrates in

Table 3 First heating endothermic DSC results of PLA

\begin{tabular}{|c|c|c|c|c|c|}
\hline Sample & $T_{\text {cc }}$, peak $\left({ }^{\circ} \mathrm{C}\right)$ & $T_{\mathrm{m}}$, peak $\left({ }^{\circ} \mathrm{C}\right)$ & $\Delta H_{\mathrm{cc}}\left(\mathrm{J} \mathrm{g}^{-1}\right.$ PLA $)$ & $\Delta H_{\mathrm{m}}\left(\mathrm{J} \mathrm{g}^{-1}\right.$ PLA $)$ & $X_{\mathrm{c}}(\%)$ \\
\hline PLA & 108 & 167 & 27.3 & 33.6 & 7.4 \\
\hline 95PLA/5EGMA & 96 & 165 & 28.7 & 35.8 & 8.0 \\
\hline 95PLA/5EGMA/0.2DCP & 95 & 165 & 28.7 & 36.7 & 8.9 \\
\hline
\end{tabular}



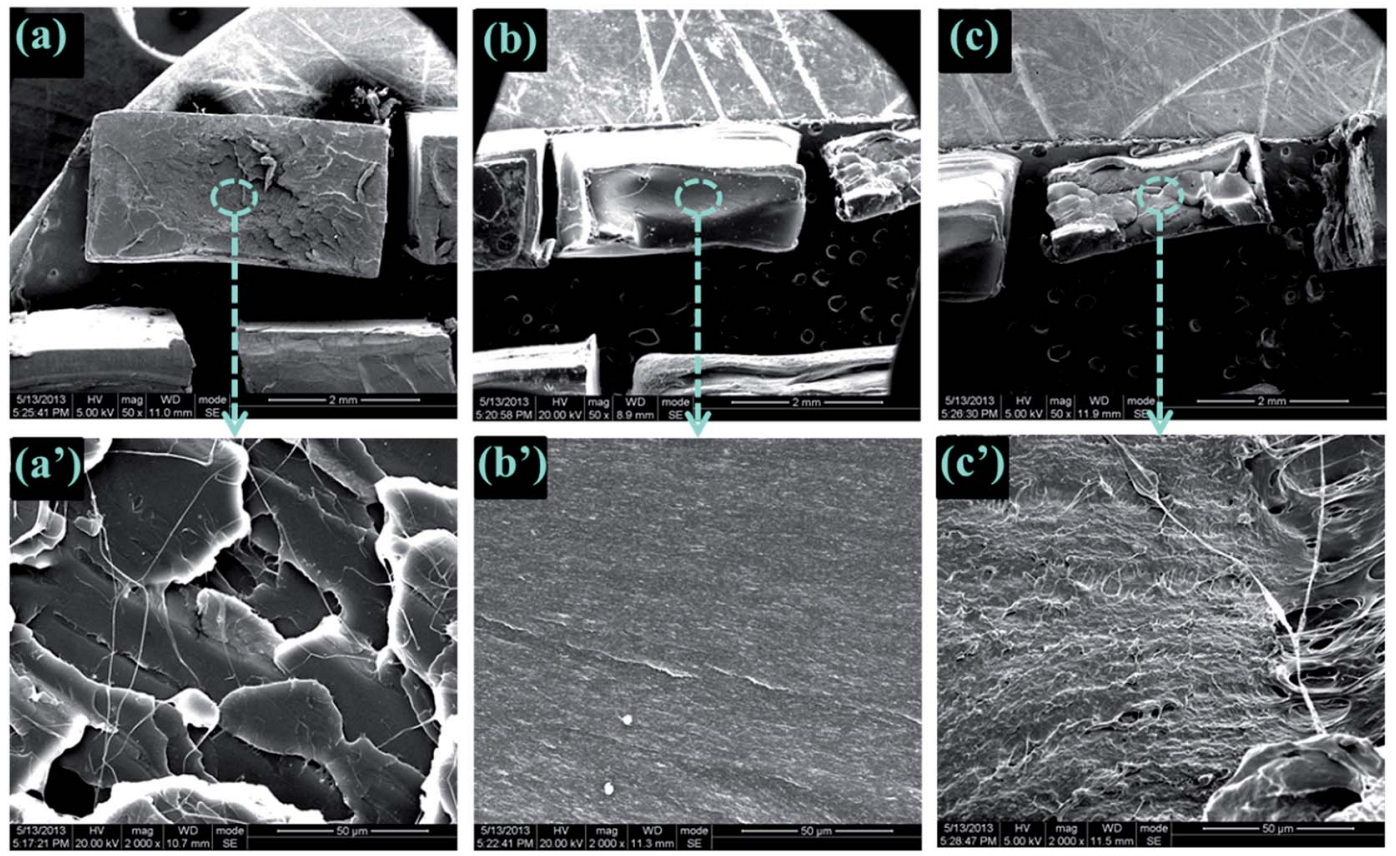

Fig. 6 SEM micrographs of the tensile fracture surface for neat PLA ((a) and (a')), 95PLA/5EGMA ((b) and (b')) and 95PLA/5EGMA/0.1DCP ((c) and $\left.\left(c^{\prime}\right)\right)$.

Fig. $6\left(c^{\prime}\right)$ that extensive plastic deformation occurs during stretching for the blend of 95PLA/5EGMA/0.1DCP, and numerous microvoids on the tensile fracture surface are observed in the meantime. It is generally accepted that, despite cavitation of elastomer particles dose contribute to the dissipation of fracture energy, the cavitated particles can relax the locally triaxial stress and then initiate crazing and/or matrix shear yielding around them. Matrix shear yielding is more effective energy dissipation fashion, compared to crazing. This indicates that 95PLA/5EGMA/0.1DCP blend undergo ductile fracture through a matrix shear yielding mechanism, which is the typical energy absorption mechanism for elastomer toughed materials. In many cases, ${ }^{38,39}$ micro-voiding either by cavitation or debonding was the essential step to trigger matrix shear yielding. SEM analysis of the sub-fracture surface, which was cryo-fractured longitudinally, was further employed to identify the micromechanical deformation process of the above blends. As shown in Fig. 7(a), a large number of black holes due to the debonding of the EGMA particles are observed for 95PLA/ 5EGMA blend. It is proposed that ordinary interfacial adhesion between EMGA and PLA matrix can easily cause the debonding between PLA matrix and EGMA particles during stretching, and ultimately result in unstable cracking. For 95PLA/5EGMA/0.1DCP blend, extensive voiding inside the occluded elastomer particles is observed. This can be understood as the fact that the enhanced interfacial adhesion, which is ascribed from the reactive compatibilization of DCP, can prevent the debonding and facilitate the cavitation of EGMA particles during stretching. The cavitation of EGMA particles can trigger the matrix shear yielding deformation, which results in extensive energy dissipation and significant improvement of flexibility.
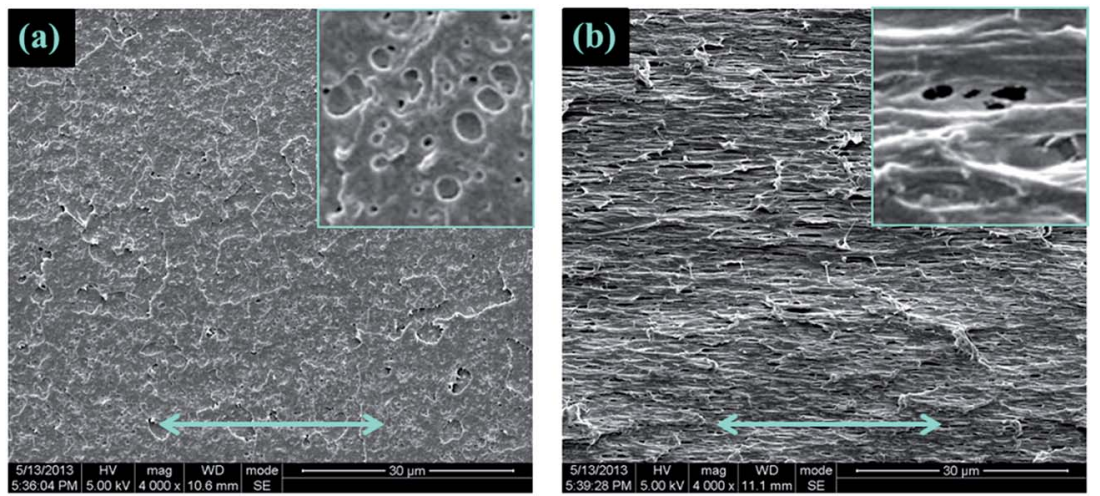

Fig. 7 SEM micrographs of the necked regions (underneath the tensile fracture) after stretching: (a) 95PLA/5EGMA, (b) 95PLA/5EGMA/0.1DCP. 


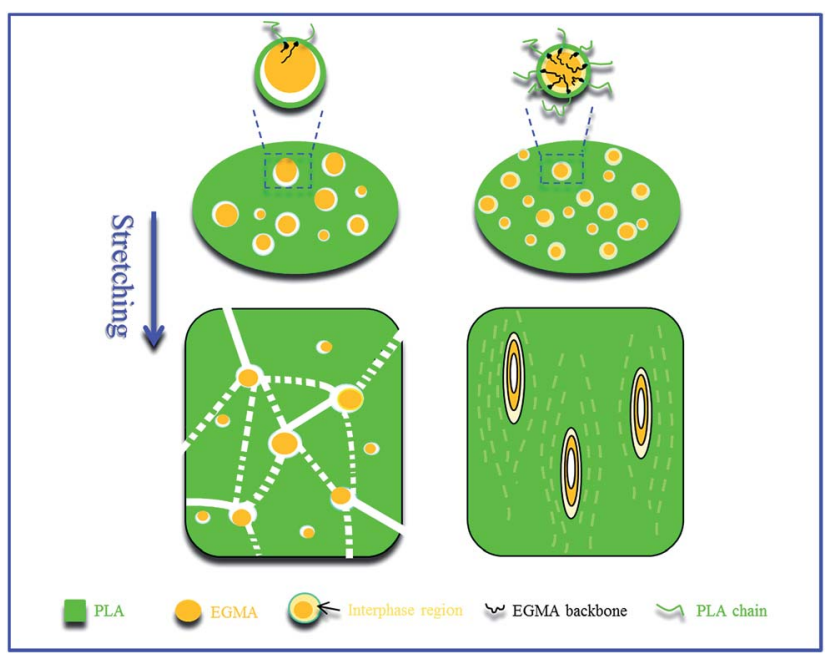

Fig. 8 Schematic representations showing the toughening mechanism difference.

From the above analysis, the dramatically improved flexibility of PLA/EGMA blends by using DCP can be mainly ascribed to the combination of the following two facts: (1) stronger interfacial adhesion between PLA and EGMA obtained by reactive interfacial compatiblization; (2) significantly decreased diameter of the EGMA particles which could be uniformly dispersed in the PLA matrix. As shown in Fig. 8, sufficient interfacial adhesion is needed to prevent the cracks initiated at the interface from growing to disaster failure and facilitate load transfer between PLA and EGMA, thus inducing cavitation of EGMA particles and matrix shear yielding deformation, which can trigger extensive energy dissipation and superior flexibility.

\subsection{Optical properties}

The optical transparence of heterogeneous polymeric materials has attracted much attention, especially for the use of package materials. Fig. 9(a) shows the quantitative ultraviolet-visible light (UV-vis) transmittance spectra of the neat PLA and its blends. Neat PLA exhibits a very high transmittance of $\sim 80 \%$ above a wavelength of $450 \mathrm{~nm}$. It is clearly shown that 95PLA/ 5EGMA blend exhibits a slightly decreased transmittance over the entire spectrum region, compared with the pure PLA. As shown in Fig. 9(b), for the light with a wavelength of $550 \mathrm{~nm}$, transmittance of neat PLA and 95PLA/5EGMA blend is $83 \%$ and $75 \%$, respectively, indicating the high clearance of the prepared 95PLA/5EGMA blend. This is most probably associated with the fact that low content of EGMA particles yielded less light scattering cross-section, namely, producing a small number of optical interfaces in the blends which can cause significant loss of transmitted light, resulting in slight lower transmittance. However, there is an evident increase of transmittance of PLA/ EGMA/DCP blend over the entire wavelength region, compared with that of PLA/EGMA blend. Besides, the transmittance of PLA/EGMA/DCP blend is improved with the increasing content of DCP, and 95PLA/5EGMA/0.2DCP blend exhibits the similar transmittance as about $82 \%$ as that of pure PLA, for the light with a wavelength of $550 \mathrm{~nm}$ (Fig. 9(b)). According to the Rayleigh Scattering Law, both the relative size of the dispersed phase to the wavelength and the difference in the refractive index of the two phases have an effect on the scattering loss in a heterogeneous system..$^{\mathbf{2 0}, 40}$ Hence, the size of the dispersed phase is a crucial factor in reducing the scattering loss when the refractive indexes of the two components are different. From above SEM analysis, the average diameter of EGMA particles is in the sub micro scale for the PLA/EGMA and PLA/EGMA/DCP blends, and the relative size of EGMA particles in PLA/EGMA/DCP blend is much smaller than that of PLA/ EGMA blend. This is associated with good optical transparence of PLA/EGMA blend and the much better optical transparence of PLA/EGMA/DCP blend. For semi-crystalline polymers, the crystallinity is another critical factor would influence the transparency, and in our work, the extremely low crystallinity maybe another favorable factor for the good transparency. PLA/EGMA/DCP blend, which has good balance of flexibility and stiffness, might be widely used in daily life, especially as packaging materials when the clearance property is needed.

\section{Conclusions}

PLA materials with excellent comprehensive mechanical performance are successfully prepared by introducing a small content of initiator DCP into the PLA/EGMA blend. The
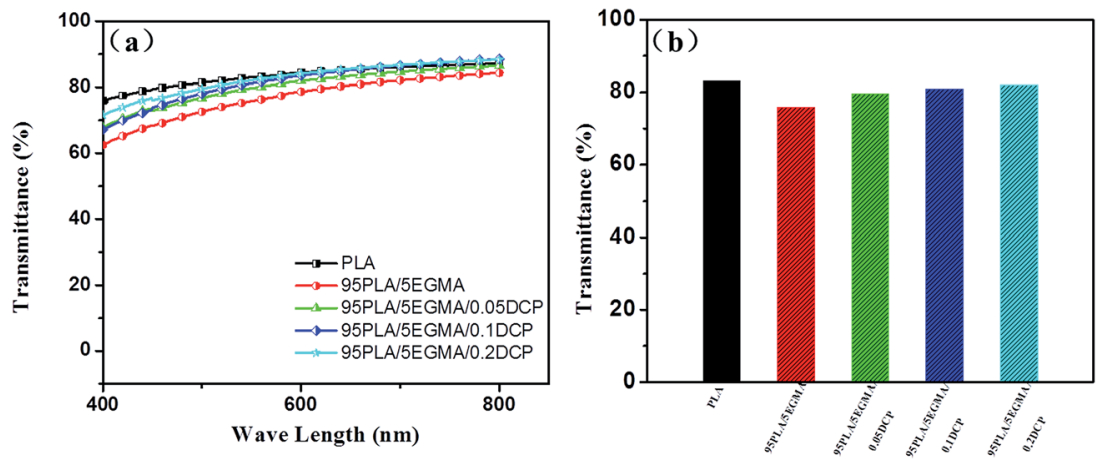

Fig. 9 Optical properties of neat PLA and its blends: (a) UV-vis transmittance spectra, (b) transmittance at a wavelength of $550 \mathrm{~nm}$. 
elongation at break of PLA/EGMA/DCP blends is about 58 times higher than that of pure PLA without sacrificing its stiffness. The highly improved toughness could be mainly ascribed to the reactive compatibilization of DCP, resulting in strong interfacial interaction of PLA/EGMA and good dispersion of EGMA particles in PLA matrix. These could effectively induce the cavitation of EGMA particles during stretching and trigger the shear yielding deformation of PLA matrix, leading to extensive energy dissipation and superior tensile toughness.

\section{Acknowledgements}

The Project Supported by Zhejiang Provincial Natural Science Foundation of China (LQ16E030005) is gratefully appreciated.

\section{References}

1 M. M. Reddy, S. Vivekanandhan, M. Misra, S. K. Bhatia and A. K. Mohanty, Prog. Polym. Sci., 2013, 38, 1653.

2 J. L. Tschan, E. Brulé, P. Haquette and C. M. Thomas, Polym. Chem., 2011, 3, 836.

3 Y. K. An, S. D. Jiang, S. K. Yan, J. R. Sun and X. S. Chen, Chin. J. Polym. Sci., 2011, 29, 513.

4 E. Fortunati, I. Armentano, Q. Zhou, D. Puglia, A. Terenzi, L. A. Berglund and J. M. Kenny, Polym. Degrad. Stab., 2012, 45, 2027.

5 Q. Zhang, V. N. Mochalin, I. Neitzel, K. Hazeli, J. Niu, A. Kontsos, J. G. Zhou, P. I. Leikes and Y. Gogotsi, Biomaterials, 2012, 33, 5067.

6 J. W. Rhim, H. M. Park and C. S. Ha, Prog. Polym. Sci., 2013, 38, 1629.

7 R. M. Rasal, A. V. Janorkar and D. E. Hirt, Prog. Polym. Sci., 2010, 35, 338.

8 Y. Li and H. Shimizu, Eur. Polym. J., 2009, 45, 738.

9 L. Jiang, J. Zhang and M. P. Wolcott, Polymer, 2007, 48, 7632.

10 Y. Y. Shi, Y. L. Li, J. Wu, T. Huang, C. Chen, Y. Peng and Y. Wang, J. Polym. Sci., Part B: Polym. Phys., 2011, 49, 267.

11 L. Jiang, M. P. Wolcott and J. Zhang, Biomacromolecules, 2006, 7, 199.

12 R. Wang, S. Wang, Y. Zhang, C. Y. Wan and P. M. Ma, Polym. Eng. Sci., 2009, 49, 2.

13 M. Harada, K. Iida, K. Okamoto, H. Hayashi and K. Hirano, Polym. Eng. Sci., 2008, 48, 1359.

14 X. F. Wang, Z. X. Zhang, J. L. Li, J. H. Yang, Y. Wang and J. H. Zhang, RSC Adv, 2015, 5, 22.

15 T. H. Zhao, Y. He, Y. D. Li, M. Wang and J. B. Zeng, RSC Adv, 2016, 6, 79542 .
16 G. C. Liu, Y. S. He, J. B. Zeng, Y. Xu and Y. Z. Wang, Polym. Chem., 2014, 5, 2530.

17 H. T. Oyama, Polymer, 2009, 50, 747.

18 H. Z. Liu, F. Chen, B. Liu, G. Estep and J. W. Zhang, Macromolecules, 2010, 43, 6058.

19 H. Z. Liu, W. J. Song, F. Chen, L. Guo and J. W. Zhang, Macromolecules, 2011, 44, 1513.

20 W. Dong, F. H. Jiang, L. P. Zhao, J. C. You, X. J. Cao and Y. J. Li, ACS Appl. Mater. Interfaces, 2012, 4, 3667.

21 J. B. Zeng, K. A. Li and A. K. Du, $R S C A d v, 2015,15,4260$.

22 G. C. Liu, Y. S. He, J. B. Zeng, Q. T. Li and Y. Z. Wang, Biomacromolecules, 2014, 10, 32546.

23 Z. Wei, C. Long and Z. Yu, Polymer, 2009, 50, 1311.

24 J. D. Jiang, L. L. Su, K. Zhang and G. Z. J. Wu, Appl. Polym. Sci., 2013, 128, 3993.

25 C. B. Bucknall and D. R. Paul, Polymer, 2009, 50, 5539.

26 A. Galeski and Z. Bartczak, Macromol. Symp., 2003, 194, 47.

27 R. A. C. Deblieck, D. J. M. van Beek, K. Remerie and I. M. Ward, Polymer, 2011, 52(14), 2979-2990.

28 L. Liu, J. Hou, L. Wang, J. Zhang and Y. Duan, Ind. Eng. Chem. Res., 2016, 55, 9907.

29 T. Semba, K. Kitagawa, U. S. Ishiaku, M. Kotaki and H. Hamada, J. Appl. Polym. Sci., 2007, 103, 1066.

30 T. Semba, K. Kitagawa, U. S. Ishiaku and H. Hamada, J. Appl. Polym. Sci., 2006, 101, 816.

31 A. Benhamida, M. Kaci, S. Cimmino, C. Silvestre and D. Duraccio, Macromol. Mater. Eng., 2009, 294, 122.

32 T. Semba, K. Kitagawa, U. S. Ishiaku and H. J. Hamada, Appl. Polym. Sci., 2006, 101, 1816-1825.

33 R. Al-Itry, K. Lamnawar and A. Maazouz, Rheol. Acta, 2014, 53, 501.

34 M. M. Mazidi and M. K. R. Aghjeh, Polym. Bull., 2015, 72, 1975.

35 K. Shahbazi, M. K. R. Aghjeh, F. Abbasi, P. Meran and M. M. Mazidi, Polym. Bull., 2012, 69, 241.

36 G. Basseri, M. M. Mazidi, F. Hosseini and M. K. R. Aghjeh, Polym. Bull., 2014, 71, 465.

37 H. W. Bai, H. Xiu, J. Gao, H. Deng, Q. Zhang, M. B. Yang and Q. Fu, ACS Appl. Mater. Interfaces, 2012, 4, 897.

38 Q. Zhao, Y. Ding, B. Yang, N. Ning and Q. Fu, Polym. Test., 2013, 32, 299.

39 Y. Li and H. Shimizu, Macromol. Biosci., 2007, 7, 921.

40 F. B. Bohren and D. R. Huffman, Absorption and Scattering of Light by Small Particles, John Wiley \& Sons, Inc., New York, 1983. 